\title{
Rapid detection of human infections with fluorine-18 fluorodeoxyglucose and positron emission tomography: preliminary results
}

\author{
Yoshifumi Sugawara1 ${ }^{1}$, Daniel K. Braun²*, Paul V. Kison ${ }^{1}$, Joseph E. Russo ${ }^{1}$, Kenneth R. Zasadny ${ }^{1}$, Richard L. Wahl1, 3 \\ 1 Division of Nuclear Medicine, The University of Michigan Medical Center, Ann Arbor, Michigan, USA \\ 2 Department of Internal Medicine, The University of Michigan Medical Center, Ann Arbor, Michigan, USA \\ 3 Department of Radiology, The University of Michigan Medical Center, Ann Arbor, Michigan, USA
}

Received 5 March and in revised form 20 May 1998

\begin{abstract}
The purpose of this study was to evaluate the feasibility of 2-[fluorine-18]fluoro-2-deoxy-D-glucose (FDG) and positron emission tomography (PET) for rapid detection of human infections. Eleven patients who were known or suspected to be harboring various infections were studied with FDG-PET. Dynamic scans over the putative infection sites were performed immediately after FDG $(370 \mathrm{MBq})$ injection through $60 \mathrm{~min}$, and static images including multiple projection images were then obtained. FDG uptake was assessed visually into four grades $(0$, normal; 1 , probably normal; 2 , probably abnormal; 3, definitely abnormal). For the semiquantitative index of FDG uptake in infections, the standardized uptake value of FDG normalized to the predicted lean body mass (SUV-lean, SUL) was determined from the images obtained at 50-60 min after FDG injection. PET results were compared with final clinical diagnoses. Eleven lesions in eight patients, which were interpreted as grade 2 or 3 by FDG-PET, were all concordant with active infectious foci. The SUL values of infections ranged from 0.97 to 6.69 . In two patients, FDG-PET correctly showed no active infection. In one patient, it was difficult to detect infectious foci by FDG-PET due to substantial normal background uptake of FDG. In total, FDG-PET correctly diagnosed the presence or absence of active infection in 10 of 11 patients. Fusion images of PET with computed tomography showed the most intense FDG uptake to be within an abscess wall. In conclusion, FDG-PET appears to be a promising modality for rapid imaging of active human infections. More extensive clinical evaluation is warranted to determine the accuracy of this method.
\end{abstract}

\footnotetext{
* Current address: Infectious Diseases Therapeutics, Drop Code 2133, Lilly Research Laboratories, Eli Lilly \& Co., Indianapolis, Indiana, USA

Correspondence to: R.L. Wahl, Division of Nuclear Medicine, University of Michigan Medical Center, 1500 E. Medical Center Dr., B1G 505 C, Ann Arbor, MI 48109-0028, USA
}

Key words: Fluorine-18 fluorodeoxyglucose - Positron emission tomography - Infection

Eur J Nucl Med (1998) 25:1238-1243

\section{Introduction}

2-[fluorine-18]fluoro-2-deoxy-D-glucose (FDG) has been shown to accumulate in malignant tumors due to their increased glucose metabolism [1]. Recently FDG has been used extensively with positron emission tomography (PET) for differentiating malignant from benign tumors, for tumor staging, and for evaluating treatment efficacy in cancer patients [2].

On the other hand, it has also been reported that activated leukocytes and macrophages have increased glucose utilization in vitro [3]. In vivo, increased FDG uptake has been shown in rodents with experimental infections and inflammatory processes [4-6]. In humans, since Tahara et al. [7] first demonstrated high FDG uptake in human abdominal abscess by PET, there have been several reports that FDG accumulates in some inflammatory lesions such as brain abscess [8-10], pulmonary granuloma [11], tuberculosis [12], and sarcoidosis [13]. However, these are generally isolated case reports and the performance of FDG-PET in infections is still not fully elucidated.

For the evaluation of infections, gallium-67 citrate $\left({ }^{67} \mathrm{Ga}\right)$ and indium-111-labeled white blood cell $\left({ }^{111} \mathrm{In}-\right.$ WBC) have generally been applied in clinical practice $[14,15] .{ }^{67} \mathrm{Ga}$ or ${ }^{111} \mathrm{In}-\mathrm{WBC}$ localization to infections has been reported within $4-6 \mathrm{~h}$ post injection, but clinical images are more commonly obtained at 24-72 h after tracer injections. Disadvantages of imaging with these tracers, including the considerable inconvenience and expense of cell labeling, are also well recognized [16, 17]. If FDG accumulates rapidly in human infectious fo- 
ci, it may have practical clinical utility as an infection detection agent.

In this study, we preliminarily evaluated the feasibility of the FDG-PET approach in 11 patients with a variety of known or suspected infections of bone and soft tissue, abdominal, and paranasal sinus regions. PET results were compared with those of other scintigraphic modalities including ${ }^{111} \mathrm{In}-\mathrm{WBC},{ }^{67} \mathrm{Ga}$ and technetium$99 \mathrm{~m}$ methylene diphosphonate (99mTc-MDP) bone scintigraphy, as well as the final clinical diagnosis based on all available data except for FDG-PET. In one case, we also evaluated infectious foci and their extent by creating fusion images of FDG-PET with computed tomography (CT).

\section{Materials and methods}

Patient population. Eleven patients, eight males and three females, aged 20-79 years, with (or suspected of harboring) various infections were studied with FDG-PET (Table 1). To be eligible, patients had to have a clinical suspicion of infection and scintigraphic, other radiological evidence, or culture evidence to confirm the presence or absence of infection (bone and soft tissue eight, abdomen in two, and paranasal sinus in one). FDG-PET results were not made available for clinical management decisions. All patients provided written informed consent for this study, which was approved by the institutional review board and conducted under the guidelines for a physician-sponsored investigational new drug. Patients were fasted for at least $4 \mathrm{~h}$ before FDG-PET study, except for one patient (patient 4) who inadvertently consumed some juice about $1 \mathrm{~h}$ before PET. The serum glucose levels during the FDGPET study were measured in nine patients, the exceptions being patients 3 and 7, in whom such measurements were not performed due to technical difficulties. Four patients had diabetes mellitus (patients 3, 5, 6, 8). All patients had received some antibiotic therapy by the time of FDG-PET study. Abscess drainage was performed in two cases (patients 8,9), amputation was performed after conservative therapy in two cases (patient 3,5 ) and necropsy was finally obtained in one case (patient 10).

FDG-PET study. FDG was produced by nucleophilic fluorination as previously described [18]. Two types of Siemens PET scanner (Siemens Medical Systems, Iselin, N.J.) were used in this study: a model 931 ECAT (15 scanning planes, 10-cm longitudinal field of view) and a model 921 EXACT (47 scanning planes, 15-cm longitudinal field of view). The reconstructed $x-y$ resolution with the Hanning filter cut-off value of 0.3 was approximately $12 \mathrm{~mm}$ for both scanners. Before tracer injection, at least one 10-min transmission scan was obtained at the level of the suspected infectious lesion using a germanium-68 ring or rod source for the purpose of attenuation correction of the emission images. Sequential dynamic scans over the infectious lesions were obtained immediately after intravenous (i.v.) tracer (370 $\mathrm{MBq}$ of FDG) injection through $60 \mathrm{~min}$ post injection, and then static scans, including multiple levels for making projection images, were obtained using 2-10 min acquisition time per scan level according to the patient status and field of view imaged. The dynamic scan of one case (patient 4) and the static scans of two cases (patients 9,11) were not obtained due to patient discomfort or refusal.

Correlative imaging studies. All patients were evaluated with one or more other correlative imaging modalities, including conventional radiography, CT, and various types of scintigraphy including ${ }^{99 \mathrm{~m} T c-M D P},{ }^{111} \mathrm{In}-\mathrm{WBC}$, and ${ }^{67} \mathrm{Ga}$ as was viewed clinically appropriate. For bone scintigraphy, following the i.v. injection of $925 \mathrm{MBq}(25 \mathrm{mCi})$ of $99 \mathrm{mTc}-\mathrm{MDP}$, immediate sequential blood flow and blood pool images were obtained over the infectious le-

Table 1. Patient populations and characteristics

\begin{tabular}{|c|c|c|c|c|c|c|c|c|c|}
\hline \multirow{2}{*}{$\begin{array}{l}\text { Patient } \\
\text { no. }\end{array}$} & \multirow[t]{2}{*}{ Age } & \multirow[t]{2}{*}{ Sex } & \multirow[t]{2}{*}{ Infection } & \multirow[t]{2}{*}{ Location } & \multicolumn{2}{|c|}{ FDG-PET } & \multirow{2}{*}{$\begin{array}{l}\text { Serum } \\
\text { glucose } \\
(\mathrm{mg} / \mathrm{dl})\end{array}$} & \multicolumn{2}{|l|}{ Corresponding imaging } \\
\hline & & & & & Grade & SUL (Ratio*) & & Modality & Grade \\
\hline 1 & 28 & $\mathrm{~m}$ & Osteomyelitis & L knee & 3 & $4.63(5.65)$ & 80 & 99mTc-MDP, ${ }^{111} \mathrm{In}-\mathrm{WBC}$ & 3,3 \\
\hline 2 & 20 & $\mathrm{~m}$ & Osteomyelitis & $\mathrm{R}$ tibia & 2 & NA & 134 & 99mTc-MDP, ${ }^{111} \mathrm{In}-\mathrm{WBC}$ & 2,3 \\
\hline \multirow[t]{2}{*}{3} & 59 & $\mathrm{~m}$ & Osteomyelitis & L ankle & 3 & $2.31(1.72)$ & NA & 99mTc-MDP & 2 \\
\hline & & & & $\mathrm{R}$ heel & 2 & NA & & 99mTc-MDP & 2 \\
\hline 4 & 24 & $\mathrm{~m}$ & $\begin{array}{l}\text { Osteomyelitis } \\
\text { (chronic) }\end{array}$ & $\mathrm{R}$ femur & 1 & NA & 71 & 99mTc-MDP & 3 \\
\hline 5 & 39 & $\mathrm{~m}$ & Osteomyelitis & $\mathrm{R}$ foot stump & 3 & $1.35(1.71)$ & 88 & 99mTc-MDP, ${ }^{111} \mathrm{In}-\mathrm{WBC}$ & 3,3 \\
\hline \multirow[t]{2}{*}{6} & 77 & $\mathrm{~m}$ & Cellulitis with & L calf & 3 & NA & 64 & 99mTc-MDP & 2 \\
\hline & & & osteomyelitis & $\mathrm{L}$ toe & 3 & $1.59(1.41)$ & & 99mTc-MDP & 3 \\
\hline 7 & 77 & $\mathrm{f}$ & Cellulitis & L ankle & 3 & $0.97(1.52)$ & NA & 99mTc-MDP & 2 \\
\hline 8 & 60 & $\mathrm{~m}$ & L inguinal abscess & L inguinal & 3 & $6.69(5.53)$ & 114 & $\mathrm{CT},{ }^{67} \mathrm{Ga}$ & 3,3 \\
\hline \multirow[t]{2}{*}{9} & 79 & $\mathrm{f}$ & Subphrenic abscess & Subphrenic & 2 & $5.54(2.32)$ & 120 & $\mathrm{CT},{ }^{67} \mathrm{Ga}$ & 3,2 \\
\hline & & & & Subphrenic & 2 & $5.30(2.22)$ & & $\mathrm{CT},{ }^{67} \mathrm{Ga}$ & 3, NA \\
\hline \multirow[t]{2}{*}{10} & 58 & $\mathrm{~m}$ & Sepsis & Abdominal wall & 1 & $1.98(1.75)$ & 300 & $\mathrm{CT},{ }^{111} \mathrm{In}-\mathrm{WBC}$ & 2,2 \\
\hline & & & (unknown origin $^{a}$ ) & Pelvic cavity & 0 & NA & & $\mathrm{CT},{ }^{111} \mathrm{In}-\mathrm{WBC}$ & 1,2 \\
\hline 11 & 41 & $\mathrm{f}$ & $\begin{array}{l}\text { Sinusitis } \\
\text { (chronic) }\end{array}$ & Paranasal sinus & 0 & NA & 96 & $\mathrm{CT},{ }^{111} \mathrm{In}-\mathrm{WBC},{ }^{67} \mathrm{Ga}$ & $1,0,0$ \\
\hline
\end{tabular}

SUL, standardized uptake value lean; Ratio*, SUL ratio of infectious lesion to surrounding normal tissue; m, male; f, female; R, right; L, left; NA, not available

a Necropsy reported the sepsis to be due to necrotizing fasciitis of abdominal wall 
sions, followed by static delayed images of the entire skeleton at $3 \mathrm{~h}$ post injection (three-phase study). ${ }^{111} \mathrm{In}-\mathrm{WBC}$ scans of the whole body and planar images with several spot views were obtained at $24 \mathrm{~h}$ after the i.v. injections of $18.5 \mathrm{MBq}(0.5 \mathrm{mCi})$ of autologous ${ }^{111} \mathrm{In}$ oxine-labeled leukocytes. ${ }^{67} \mathrm{Ga}$ scans of whole body and planar images with several spot views were also obtained at 24-72 h after the i.v. injection of $370 \mathrm{MBq}(10 \mathrm{mCi})$ of ${ }^{67} \mathrm{Ga}$ citrate. In one patient (patient 8 ), fusion images were made by combining the CT and PET images on a computer workstation (Sparcstation II; Sun Microsystems, MountainView, Calif.) with an automatic mutual information-based registration algorithm, as recently reported $[19,20]$. Based on all clinical data except for FDG-PET, a clinical diagnosis was established by the referring physician, and this was used for comparison with the PET results.
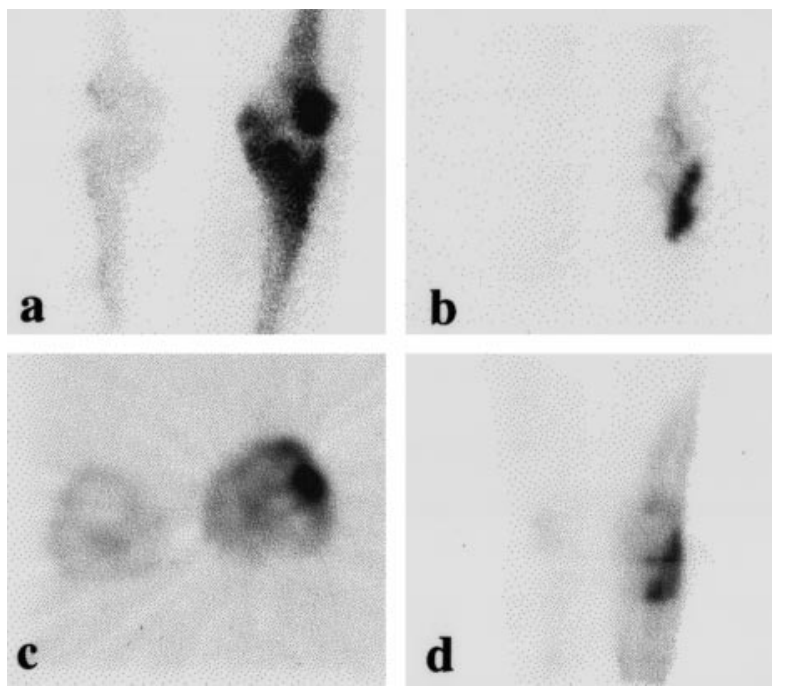

Fig. 1a-d. A 28-year-old male (patient no. 1) with a history of multiple fractures around the left knee, presented with clinical symptoms consistent with active osteomyelitis. a $99 \mathrm{~m}$ Tc-MDP bone scan, $\mathbf{b}{ }^{111}$ In-WBC scan, $\mathbf{c}$ transverse FDG-PET image and $\mathbf{d}$ FDG-PET projection image; images show focal and intense uptake at the left knee consistent with active osteomyelitis. Note that the FDG distribution is not identical to that of the bone scanning agent due to the different physiologies of localization
Image interpretation. The FDG uptake in the last (50-60 min) frame of the dynamic scans and static scans (including projection images) was assessed visually and the degree of abnormality was classified into four grades $(0$, normal; 1 , probably normal; 2 , probably abnormal; 3, definitely abnormal) by two independent observers (Y.S., R.L.W.) who were blinded to the results of other imaging studies and final diagnoses. If there were any disparities between their independent gradings of the lesions, consensus interpretations were achieved after review of the images and discussion. For a semiquantitative index of FDG uptake in sites of infection, the last (50-60 min) frame of the dynamic scans was used to define regions of interest (ROIs). To determine the maximal FDG uptake in areas believed to represent infectious foci and minimize the influence of partial volume effects (and thus better assure accurate signal recovery), a small square ROI ( $4 \times 4$ pixels) was placed by means of an automated algorithm on the maximal area of FDG uptake within a larger ROI covering the infectious lesions. The standardized uptake value (SUV) calculated on the basis of predicted lean body mass (SUV-lean, SUL) was determined in this maximal uptake ROI, as previously described [21]. The average uptake of FDG in the surrounding (or opposite) normal tissue was determined by means of irregular ROIs.

\section{Results}

Eleven lesions in eight patients (patients 1-3, 5-9), which were visually interpreted as grade 2 or 3 by FDGPET, were all concordant with active infectious foci (Table 1). The maximal SUL values of infectious lesions ranged from 0.97 to 6.69 and the SUL ratios of infections to surrounding normal tissues ranged from 1.41 to

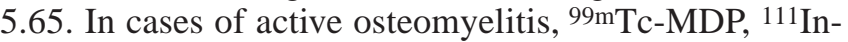
WBC, and FDG-PET images all showed obvious intense uptake in the infectious foci, while the FDG distribution was more focal than that of $99 \mathrm{mTc}-\mathrm{MDP}$ and concordant with the ${ }^{111}$ In-WBC image (Fig. 1). In a case of cellulitis, FDG-PET clearly demonstrated the infectious focus, although the early phase of triple-phase bone scans could show subtle increased uptake in the infection site (Fig. 2). In one patient (patient 8), FDG-PET showed intense ring-like FDG uptake largely concordant with the

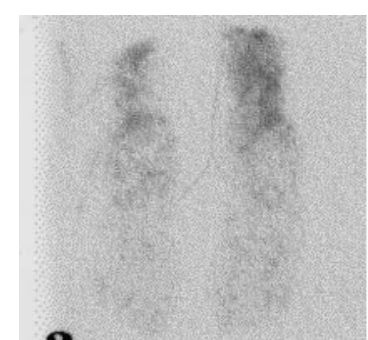

a
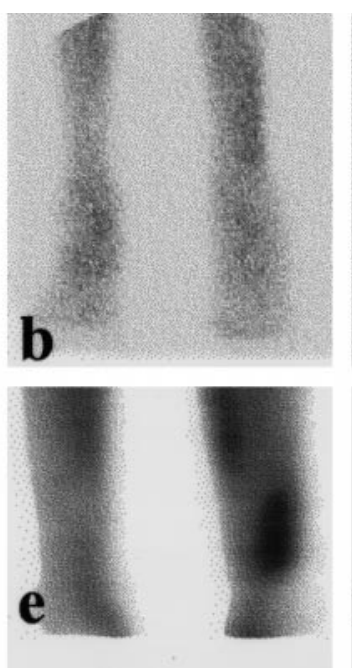
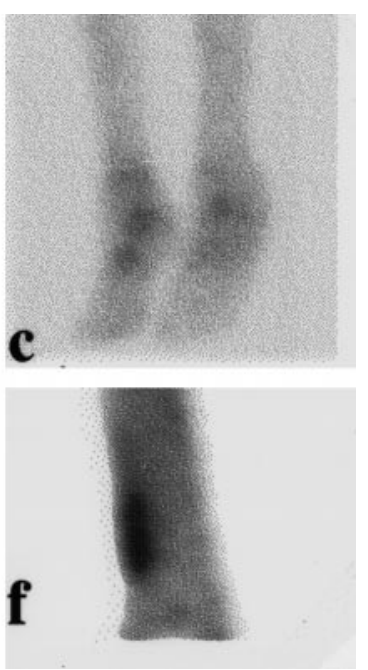

Fig. 2a-f. A 77-year-old female (patient no. 7) with chronic lymphocytic leukemia, presented with ulcers on her left lateral ankle. a Blood flow and $\mathbf{b}$ blood pool images of ${ }^{99 \mathrm{~m}} \mathrm{Tc}-\mathrm{MDP}$ bone scan show increased uptake in the left lateral ankle, but $\mathbf{c}$ delayed image shows no obvious abnormal uptake in the bone, consistent with the cellulitis but with no evidence of osteomyelitis. d Transaxial, e coronal and $\mathbf{f}$ sagittal images of FDG-PET also show intense focal uptake in the left lateral ankle consistent with cellulitis 




Fig. 3a-c. A 60-year-old male (patient no. 8) with diabetes mellitus, presented with a left inguinal abscess. a CT scan at the level of inguinal region shows an abscess cavity with ring-enhanced wall and central liquefaction in the adductor compartment of the left upper thigh. b One day after the CT scan, transverse FDGPET scan shows ring-like intense uptake of FDG in the left inguinal region. The SUL is 6.69. c A fusion image shows intense FDG uptake in the abscess which is generally localized to an enhanced abscess wall seen on CT

\section{$\mathbf{a}$}

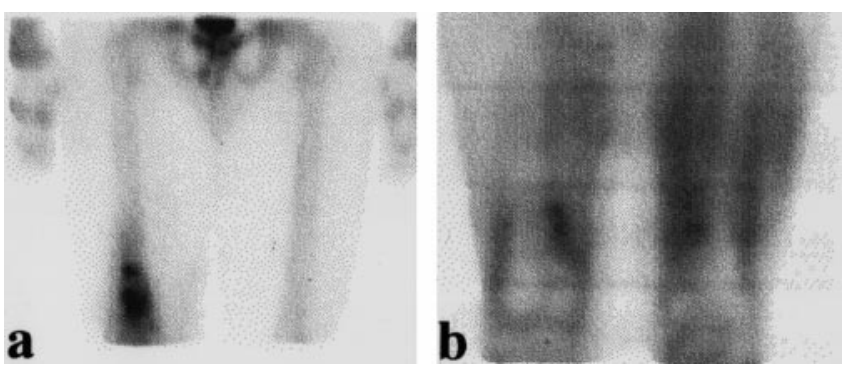

Fig. 4a,b. A 24-year-old male (patient no. 4) with chronic osteomyelitis from a previous football injury in his right distal femur. a 99mTc-MDP bone scan shows intense uptake in the right distal femoral bone. b FDG-PET projection image shows no definite abnormal bone uptake, although the image quality is impaired due to the substantial normal muscle uptake of FDG

abscess wall (SUL $=6.69)$, confirmed by fusion images with CT (Fig. 3).

On the other hand, four lesions in three patients (patients $4,10,11$ ) were interpreted as grade 1 or 0 by FDG-PET. In a case of chronic osteomyelitis (patient 4, Fig. 4), although the $99 \mathrm{~m}$ Tc-MDP bone scan remained abnormal after successful treatment, FDG-PET showed no obvious bone uptake, though the image quality was poor because of substantial muscle uptake, presumably due to insulin-mediated skeletal muscle uptake after eating. In patient 11, FDG-PET showed no abnormal uptake (grade 0), and finally no evidence of active infections was confirmed by other correlative images and clinical follow-up. It was difficult to detect infectious foci by FDG-PET in one patient (patient 10): in this patient, the FDG uptake of the abdominal wall was slightly increased but initially interpreted as a postoperative change without infection (grade 1). The image quality of FDG-PET in this case was also not optimal due to substantial increased background uptake secondary to the increased serum glucose level $(300 \mathrm{mg} / \mathrm{dl})$ and multiple prior abdominal surgical procedures. In total, FDG-PET correctly diagnosed the presence or absence of active infection in ten of 11 patients.

${ }^{111}$ In-WBC scans were performed in five patients, and five of six lesions were correctly diagnosed. However, a "false-positive" localization of tracer uptake in one patient (patient 10) was identified (this was considered to be due to uptake in Hartmann's pouch after colectomy of ischemic bowel). ${ }^{67} \mathrm{Ga}$ scans were performed in only three patients, with one lesion not detected (in patient 9, the planar ${ }^{67} \mathrm{Ga}$ image could not separate the infectious foci).

\section{Discussion}

In 1980, Som et al. [22] reported that turpentine-induced abscesses in rats showed no increased FDG uptake, while ${ }^{67} \mathrm{Ga}$ showed high uptake. They suggested that FDG could potentially differentiate malignant tumors from inflammatory lesions, while ${ }^{67} \mathrm{Ga}$ could not. However, recent experimental studies have demonstrated that FDG can accumulate in inflammatory/infectious lesions $[4-13,23,24]$. Thus, it is doubtful whether FDG can consistently differentiate malignant tumors from inflammatory lesions [25]. Indeed, FDG is not a cancer-specific agent; these characteristics of tracer uptake in infection, inflammation and tumor indicate FDG may also have clinical utility as an infection detection agent. Although it is preliminary and includes only a limited number of cases, the present study clearly demonstrates the feasibility of using FDG-PET for the detection of human infections.

Since FDG has shown rapid accumulation into infectious foci and also has a high early target to background ratio [4], FDG-PET potentially could detect infections rapidly after tracer injections. This characteristic could be of considerable benefit for patients with acute infections, because when using ${ }^{67} \mathrm{Ga}$ and ${ }^{111} \mathrm{In}-\mathrm{WBC}$ at least 4-6 h, and usually $24 \mathrm{~h}$ or more, are required after tracer injections to obtain diagnostic images. FDG shows little normal organ uptake, except for brain and heart (if the patient is not fasted), while ${ }^{67} \mathrm{Ga}$ shows high normal uptake in the liver and bowel. ${ }^{111} \mathrm{In}-\mathrm{WBC}$ also shows high 
uptake in the normal liver, spleen, and bone marrow. The radiation dose from FDG-PET is reasonably low, mainly because of the short half-life of ${ }^{18} \mathrm{~F}\left(T_{1 / 2}=\right.$ $110 \mathrm{~min})$ as compared with ${ }^{67} \mathrm{Ga}\left(T_{1 / 2}=78 \mathrm{~h}\right)$ or ${ }^{111} \mathrm{In}$ $\left(T_{1 / 2}=67 \mathrm{~h}\right)$. Moreover, PET is quantitative, with radiation attenuation by normal tissues corrected for by transmission images, which allows the determination of precise target to background uptake ratios and, with appropriate standards, the absolute fraction of injected dose reaching a tissue [26].

In cases of active osteomyelitis, ${ }^{99 m} \mathrm{Tc}-\mathrm{MDP},{ }^{67} \mathrm{Ga}$, ${ }^{111} \mathrm{In}-\mathrm{WBC}$, and FDG also showed increased tracer uptake, as typically shown in Fig. 1. As a monitor of therapy or a predictor of cure of osteomyelitis, it has been reported that ${ }^{67} \mathrm{Ga}$ and ${ }^{111} \mathrm{In}-\mathrm{WBC}$ are more sensitive than ${ }^{99 \mathrm{~m} T c-M D P}$, since ${ }^{67} \mathrm{Ga}$ and ${ }^{111} \mathrm{In}-\mathrm{WBC}$ more rapidly normalize after successful treatment whereas $99 \mathrm{mTc}$ MDP often remains abnormal for a long time [14, 17, 27]. FDG-PET also may correctly show actual infectious activity through glucose metabolism.

In cellulitis, early phase bone scans showed increased blood flow and blood pool activity, while delayed scans were normal, as previously reported [28] (and shown in Fig. 2). Indeed, the three-phase bone scan is useful for differentiating cellulitis from osteomyelitis, while it is difficult for ${ }^{67} \mathrm{Ga}$ or ${ }^{111} \mathrm{In}-\mathrm{WBC}$ (and also likely FDG) to differentiate them.

In this study, besides visual interpretation, we calculated the SUL (SUV-lean) as a weight-independent index of glucose metabolism according to the previous report [21]. The maximal SUL values of infectious lesions ranged from 0.97 to 6.69 and the SUL ratios of infectious foci to contralateral or adjacent backgrounds ranged from 1.41 to 5.65. These values were lower than those of recent reports [24] and may well have underestimated the glucose metabolism in infections, since our study included small and peripheral superficial (thin) lesions, and several diabetic patients. For example, in a case of cellulitis (patient 7, Fig. 2), a 3-cm diameter of cellulitis (indeed, this was a peripheral superficial lesion) showed focal intense FDG uptake and was interpreted as grade 3 , but the SUL in the site of infection was not very high. Thus, the lowest SUL infection was detectable in an extremity due to the minimal background uptake. For merely detecting the infectious foci, a quantitative evaluation such as SUV or SUL may well not be necessary, as previously reported in the evaluation of pulmonary abnormalities [29], although quantitation may be useful in differentiating the stages of infection and for monitoring the efficacy of treatment [24].

Moreover, the FDG uptake in infectious foci will almost certainly be affected by serum glucose levels (and also by serum insulin levels, although these were not measured in this study) and the underlying disease such as diabetes mellitus. It has been reported that both hyperglycemia and insulin-induced hypoglycemia (in previously euglycemic animals) substantially reduced FDG uptake in tumor, but conversely, relatively preserved (in hyperglycemia) or substantially increased (in insulin-induced hypoglycemia) FDG uptake in muscle and fat tissues, and as a result, tumor/muscle and tumor/fat ratios of FDG uptake significantly decreased in both conditions $[30,31]$. We expect that plasma glucose and/or insulin concentration may affect the FDG uptake in sites of infection, as reported in tumors. Thus, the FDG-PET method potentially may be of lower utility in diabetics. This may, if confirmed, represent a major limitation of the method, as diabetics are often affected with infections.

The technique of "anatometabolic" fusion imaging has been helpful in localizing foci of increased tracer activity and for staging malignant tumors [19, 32]. We made fusion images in one of our cases, which showed intense ring-like uptake of FDG in a contrast-enhancing abscess wall seen on CT (Fig. 3). Yamada et al. [6] reported that macro- and micro-autoradiography showed high density of silver grain deposition in the abscess wall consisting of an inflammatory cell layer and granulation tissue. Our observation of FDG accumulation in the abscess wall in a patient was also concordant with the result of this recent experimental study.

While FDG-PET might, on first inspection, be considered to be a "high-cost, high-tech," alternative to ${ }^{111}$ In-WBC imaging, clinical PET imaging is now a reality, with recent U.S. Medicare reimbursement of FDGPET for solitary pulmonary nodule characterization and lung cancer staging and private insurance reimbursement for PET in many European countries. In addition, the cost of FDG is likely less than that of ${ }^{111} \mathrm{In}-\mathrm{WBC}$ labeling. Additionally, lower cost methods of detecting FDG uptake, such as coincidence gamma cameras, have now become available, suggesting the FDG method may have clinical practicality-if additionally validated in larger clinical studies. Finally, if accurate diagnoses can be made using FDG-PET in $1 \mathrm{~h}$ rather than days after tracer injection, this could be cost saving.

In conclusion, this preliminary study demonstrates the feasibility of imaging human infections rapidly with FDG-PET scanning and also partly demonstrates its limitations. Accurate estimation of the sensitivity and specificity of FDG-PET will require the study of many additional patients with various kinds of infections, and follow-up studies after treatment. These initial data also show the feasibility and potential advantages of anatometabolic imaging and quantitative evaluation of infections, to better assess their extent and activity. While much more study is clearly needed, these initial data suggest FDG-PET may prove useful in the rapid detection and management of human infections.

Acknowledgements. This study was supported by the National Institute of Health grants CA53172, CA56731, CA52880 and MO1 RR00042, and the "Hi-tech funding initiative" of the University of Michigan Clinical Research Center. We thank Denise Regan for her excellent assistance in preparing data, and Charles R. Meyer, $\mathrm{MD}$, and Peter Schultz for their assistance in performing image fusion. The efforts of the PET chemistry staff and the PET imaging technologists are appreciated. 


\section{References}

1. Warburg O. The metabolism of tumors. New York Richard R. Smith; 1931: 129-169.

2. Wahl RL, Hawkins RA, Larson SM, et al. Proceedings of a National Cancer Institute workshop: PET in oncology - a clinical research agenda. Radiology 1994; 3: 604-606.

3. Fantone JC, Ward PA. Role of oxygen-derived free radicals and metabolites in leukocyte-dependent inflammatory reactions. Am J Pathol 1982; 3: 395-418.

4. Gutowski TD, Fisher SJ, Moon S, Wahl RL. Experimental studies of ${ }^{18} \mathrm{~F}-2$-fluoro-2-deoxy-D-glucose (FDG) in infection and in reactive lymph nodes [abstract]. J Nucl Med 1992; 925p.

5. Wahl RL, Fisher SJ. A comparison of FDG, L-methionine and thymidine accumulation into experimental infections and reactive lymph nodes [abstract]. J Nucl Med 1993; 104p.

6. Yamada S, Kubota K, Kubota R, Ido T, Tamahashi N. High accumulation of fluorine-18 fluorodeoxyglucose in turpentineinduced inflammatory tissue. J Nucl Med 1995; 7: 1301-1306.

7. Tahara T, Ichiya Y, Kuwabara Y, et al. High $\left[{ }^{18} \mathrm{~F}\right]$-fluorodeoxyglucose uptake in abdominal abscesses: a PET study. $J$ Comput Assist Tomogr 1989; 5: 829-831.

8. Sasaki M, Ichiya Y, Kuwabara Y, et al. Ringlike uptake of $\left[{ }^{18} \mathrm{~F}\right]$ FDG in brain abscess: a PET study. $J$ Comput Assist Tomogr 1990; 3: 486-487.

9. Hanson MW, Glantz MJ, Hoffman JM, et al. FDG-PET in the selection of brain lesions for biopsy. J Comput Assist Tomogr 1991; 5: 796-801.

10. Meyer MA, Frey KA, Schwaiger M. Discordance between F18 fluorodeoxyglucose uptake and contrast enhancement in a brain abscess. Clin Nucl Med 1993; 8: 682-684.

11. Slosman DO, Spiliopoulos A, Keller A, et al. Quantitative metabolic PET imaging of a plasma cell granuloma. $J$ Thorac Imaging 1994; 2: 116-119.

12. Patz EF Jr, Lowe VJ, Hoffman JM, et al. Focal pulmonary abnormalities: evaluation with F-18 fluorodeoxyglucose PET scanning. Radiology 1993; 2: 487-490.

13. Lewis PJ, Salama A. Uptake of fluorine-18 fluorodeoxyglucose in sarcoidosis. J Nucl Med 1994; 10: 1647-1649.

14. Alazraki NP. Gallium-67 imaging in infection. St. Louis: Mosby-Year Book; 1995: 702-713.

15. Preston DF. Indium-111 label in inflammation and neoplasm imaging. St. Louis:Mosby-Year Book; 1995: 714-724.

16. Seabold JE, Palestro CJ, Brown ML, et al. Procedure guideline for gallium scintigraphy in inflammation. Society of $\mathrm{Nu}-$ clear Medicine. J Nucl Med 1997; 6: 994-997.

17. Seabold JE, Forstrom LA, Schauwecker DS, et al. Procedure guideline for indium-111-leukocyte scintigraphy for suspected infection/inflammation. Society of Nuclear Medicine. $J$ Nucl Med 1997; 6: 997-1001.

18. Toorongian SA, Mulholland GK, Jewett DM, Bachelor MA, Kilbourn MR. Routine production of 2-deoxy-2-[ $\left.{ }^{18} \mathrm{~F}\right]$ fluoro-Dglucose by direct nucleophilic exchange on a quatenary 4-ami- nopyridinium resin. Int J Rad Appl Instrum [B] 1990; 273-279.

19. Wahl RL, Quint LE, Cieslak RD, Aisen AM, Koeppe RA, Meyer CR. "Anatometabolic" tumor imaging: fusion of FDG PET with CT or MRI to localize foci of increased activity. $J$ Nucl Med 1993; 7: 1190-1197.

20. Meyer CR, Boes JL, Kim B, et al. Demonstration of accuracy and clinical versatility of mutual information for automatic multimodality image fusion using affine and thin-plate spline warped geometric deformations. Med Image Anal 1997; 3: 195-206.

21. Zasadny KR, Wahl RL. Standardized uptake values of normal tissues at PET with 2-[fluorine-18]-fluoro-2-deoxy-D-glucose: variations with body weight and a method for correction. $R a$ diology 1993; 3: 847-850.

22. Som P, Atkins HL, Bandoypadhyay D, et al. A fluorinated glucose analog, 2-fluoro-2-deoxy-D-glucose (F-18): nontoxic tracer for rapid tumor detection. J Nucl Med 1980; 7: 670-675.

23. Palmer WE, Rosenthal DI, Schoenberg OI, et al. Quantification of inflammation in the wrist with gadolinium-enhanced MR imaging and PET with 2-[F-18]-fluoro-2-deoxy-D-glucose. Radiology 1995; 3: 647-655.

24. Ichiya Y, Kuwabara Y, Sasaki M, et al. FDG-PET in infectious lesions: The detection and assessment of lesion activity. Ann Nucl Med 1996; 2: 185-191.

25. Larson SM. Cancer or inflammation? A holy grail for nuclear medicine [editorial; comment]. J Nucl Med 1994; 10: 1653-1655.

26. Wahl RL, Cody RL, Hutchins GD, Mudgett EE. Primary and metastatic breast carcinoma: initial clinical evaluation with PET with the radiolabeled glucose analogue 2-[F-18]-fluoro2-deoxy-D-glucose. Radiology 1991; 3: 765-770.

27. Schauwecker DS. The scintigraphic diagnosis of osteomyelitis. AJR Am J Roentgenol 1992; 1: 9-18.

28. Wegener WA, Alavi A. Diagnostic imaging of musculoskeletal infection. Roentgenography; gallium, indium-labeled white blood cell, gammaglobulin, bone scintigraphy; and MRI. Orthop Clin North Am 1991; 3: 401-418.

29. Lowe VJ, Hoffman JM, DeLong DM, Patz EF, Coleman RE. Semiquantitative and visual analysis of FDG-PET images in pulmonary abnormalities. J Nucl Med 1994; 11: 1771-1776.

30. Wahl RL, Henry CA, Ethier SP. Serum glucose: effects on tumor and normal tissue accumulation of 2-[F-18]-fluoro-2-deoxy-D-glucose in rodents with mammary carcinoma. Radiology 1992; 3: 643-647.

31. Torizuka T, Fisher SJ, Wahl RL. Insulin-induced hypoglycemia decreases uptake of 2-[F-18]fluoro-2-deoxy-D-glucose into experimental mammary carcinoma. Radiology 1997; 1 : 169-172.

32. Wahl RL, Quint LE, Greenough RL, Meyer CR, White RI, Orringer MB. Staging of mediastinal non-small cell lung cancer with FDG PET, CT, and fusion images: preliminary prospective evaluation. Radiology 1994; 2: 371-377. 